\title{
Correlation of acromial morphology in association with rotator cuff tear: a retrospective study
}

\author{
Rashmeet Kaur ${ }^{B, E}$, Anshul Dahuja ${ }^{A, B, C}$, Simmi Garg ${ }^{D, E, F}$, Kapil Bansal ${ }^{D, F}$, Radhe S. Garg, ,F, Paramdeep Singh ${ }^{B, C, E}$ \\ Guru Gobind Singh Medical College and Hospital, Faridkot, Punjab, India
}

\section{Abstract}

Purpose: There have been many studies that have attempted to correlate radiographic acromial characteristics with rotator cuff tears, but the results have not been conclusive. Rotator cuff tears (RCT) are the common aetiology of shoulder pain. We assessed the association of rotator cuff tears with commonly used radiographic parameters of acromial morphology and their different radiographic characteristics.

\begin{abstract}
Material and methods: From a retrospective study of 98 patients, we characterised acromial type and measured acromial thickness (AT), critical shoulder angle (CSA), lateral acromial angle (LAA), acromiohumeral distance (AHD), and acromion index (AI) on a 1.5T MRI, from 68 patients with partial or full-thickness supraspinatus tendon tears and 30 controls without tears.
\end{abstract}

Results: Out the 68 patients with rotator cuff tear, supraspinatus was the most commonly affected tendon, with 86\% (59) cases showing abnormalities. The average age of the patients was $45.11 \pm 21.45$ years with male dominance $(80 \%)$. Partial tears of rotator cuff were more common than complete tears. Forty-eight cases showed partial tears in supraspinatus as compared to 11 cases of complete tears. The acromial type did not show any correlation with any particular cuff lesion. The AT and AI of controls were significantly smaller than cuff-tear patients. The LAA of cuff-tear patients was significantly different from that of control patients. The impingement patients demonstrated a significantly greater acromial thickness, larger CSA, decreased AHD, and decreased LAA than their control counterparts.

Conclusions: A higher prevalence of rotator cuff tears and impingement associated with low lateral acromial angle, larger CSA and decreased AHD was observed. AT and AI have a direct correlation with rotator cuff tear.

Key words: acromion, rotator cuff, supraspinatus.

\section{Introduction}

Different shapes of the acromion are associated with a variety of pathologies such as impingement syndrome and rotator cuff tear (RCT) [1]. Rotator cuff tears are one of the most common disorders of the shoulder, with recent studies emphasising the specific acromion morphologies predisposing to it. Although the specific aetiology of RCT is not fully known, it has been proposed that it results from a combination of intrinsic and extrinsic factors. The degenerative changes, hypovascularity, and micro structural collagen fibre abnormalities are the most important intrinsic factors. And the considerable extrinsic factors include subacromial impingement with chronic repetitive use and tensile overload [2]. In 1983, Neer stated that $95 \%$ of cuff tears are caused by mechanical impingement which was successfully treated by anterior acromioplasty [3]. Hence, acromioplasty proves to be the standard operative treatment for impingement lesions with a substantial increase in its incidence [4]. The accurate assessment of the extrinsic factors results in better management of the patients.

Henceforth, several classifications of the acromial morphology have been proposed, including Bigliani et al. (1986) and Kitay et al. (1995) describing the acromial slope (AS); furthermore, Kitay et al. (1995) and Aoki et al. (1986) described the acromial tilt [5-7].

\section{Correspondence address:}

Dr. Anshul Dahuja, Sadiq Rd, Kotakpura, GGS Medical Hospital, Faridkot, Punjab 151203, India, e-mail: anshuldahuja@gmail.com

Authors' contribution:

A Study design · B Data collection · C Statistical analysis · D Data interpretation · E Manuscript preparation · F Literature search · G Funds collection 
Other studies include one by Banas et al., who described the types of frontal plane slope of the acromion on magnetic resonance imaging (MRI) and found a lower lateral acromial angle (LAA) in patients with rotator cuff disease [8]. Similarly, Nyffeler et al. observed that the ac-

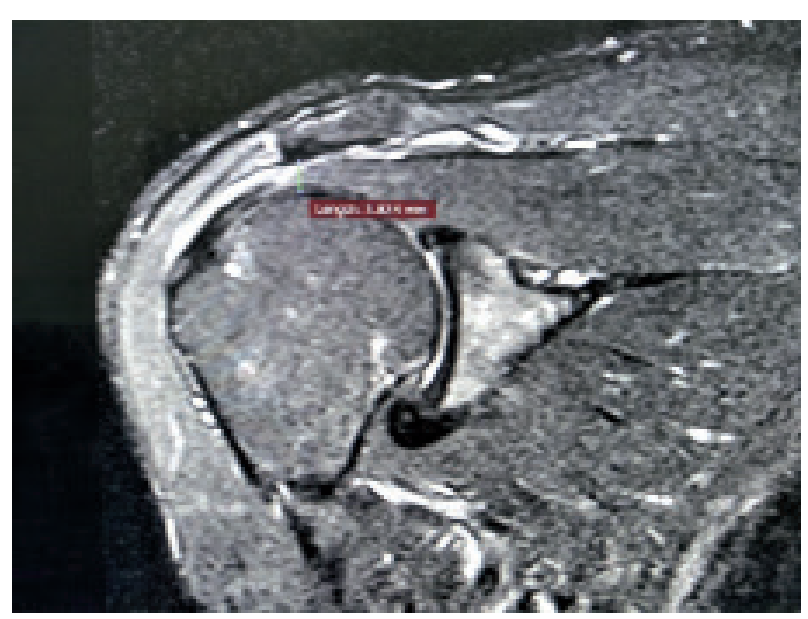

Figure 1. Acromiohumeral distance in partial rotator cuff tear

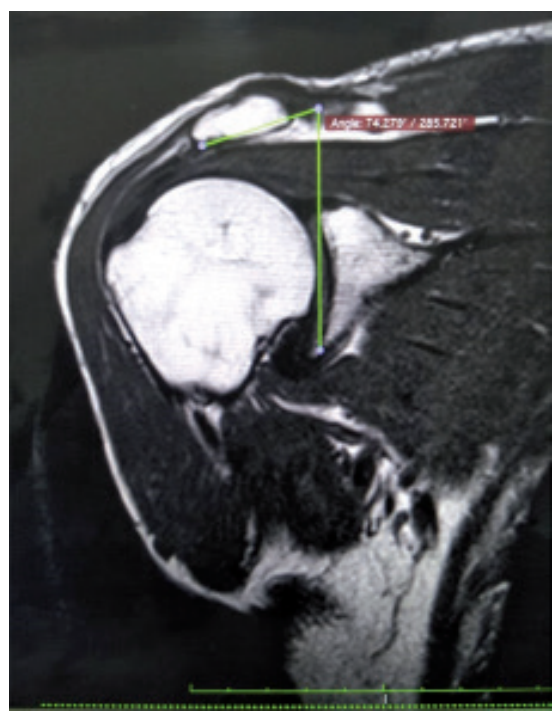

Figure 2. Lateral acromial angle

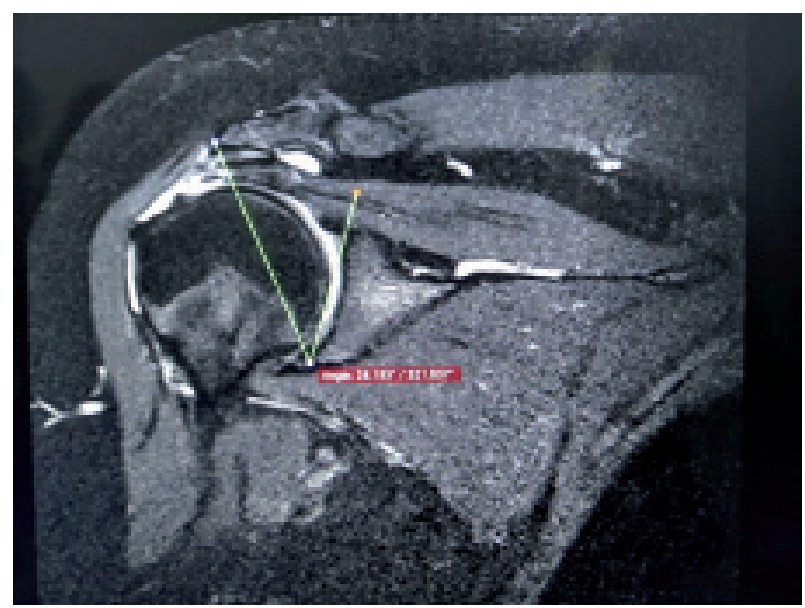

Figure 3. Critical shoulder angle romion of patients with more lateral extension had higher incidence of rotator cuff tear, and they also described the acromion index (AI) in their study [9].

In order to advance our understanding of rotator cuff disease, further investigation of the radiographic characteristics of the acromion and their potential relationships to rotator cuff disease is needed. The role of the acromion is still ambiguous, although several studies have attempted to correlate Neer's original theory of extrinsic mechanical impingement as the primary aetiological factor of rotator cuff disease. MRI has proven to be a single solution both for characterising acromial morphology and for evaluating rotator cuff tears. In this study we evaluated six commonly used parameters of acromial morphology (acromial type, acromial thickness, critical shoulder angle, lateral acromial angle, acromiohumeral distance, and acromion index) and their relationship to rotator cuff tears.

\section{Material and methods}

This study was carried out in the period from January 2015 to August 2017 in our institution and included 68 patients with RCT, either partial or full thickness (patient group). In addition, 30 subjects with an intact rotator cuff on the asymptomatic side were recruited to serve as a control group. The exclusion criteria included previous surgery, fractures and/or dislocation, infections or tumours of the shoulder, and cases with acromial spurs. All patients were imaged with MRI according to their side of complaint by a single technician, and acromial measurements were taken by a single radiologist. In order to study the incidence of each acromial shape, 30 age- and sex-matched patients without RCT were included as the control group. From a retrospective study of 98 patients, we measured acromial type (Bigliani) and acromial thickness (AT) measured on sagittal oblique images just lateral to the acromioclavicular joint as the widest part of acromion in plane perpendicular to the long axis of acromion; acromiohumeral distance (AHD) refers to the shortest distance between line parallel to the inferior edge of acromion and parallel tangent to the superior part of humeral head (as shown in Figure 1).

LAA was measured from an oblique coronal MRI slice just posterior to the acromioclavicular joint. The angle was determined by the intersection of a line parallel to the acromion undersurface and a second line parallel to the glenoid fossa (as shown in Figure 2). The critical shoulder angle (CSA) is measured between a line through the inferior lateral edge of the glenoid to the inferior lateral edge of the acromion and a line parallel to the glenoid (as shown in Figure 3) and the AI. The AI (A/B) is calculated by dividing the distance between the osseous glenoid plane to the lateral border of the acromion (A) by the distance between the osseous glenoid plane and the most lateral part of the proximal humerus, from 68 patients with partial or full-thickness supraspinatus tendon tears and 30 controls without subacromial pathology 
Table 1. Descriptive statistics

\begin{tabular}{|l|c|c|c|c|}
\hline Factor & Control group, $n=30$ & Partial RCT group, $n=57$ & Complete RCT, $n=11$ & $p$ value \\
\hline Age (years) & $46.5(39.2-66.5)$ & $42.2(34.4-58.8)$ & $57.5(41-85)$ & - \\
\hline Sex $(\mathrm{M}: \mathrm{F})$ & $28: 22$ & $46: 11$ & $7: 4$ & - \\
\hline Lateral acromial angle $\left(^{(}\right)$ & $78(67-94)$ & $72.4(67-90)$ & $69.8(63-90)$ & - \\
\hline Acromion index & $0.64(0.55-0.85)$ & $0.72(0.58-0.92)$ & $0.76(0.51-0.91)$ & $<0.001$ \\
\hline Acromiohumeral distance $(\mathrm{mm})$ & $9.4(8.5-10.9)$ & $7.8(7.1-9.4)$ & $7.1(6.4-7.9)$ & $<0.001$ \\
\hline Acromial thickness $(\mathrm{mm})$ & $7.6(7.1-8.4)$ & $8.5(7.9-9.4)$ & $8.6(7.7-9.2)$ & $<0.05$ \\
\hline Critical shoulder angle $\left(^{\circ}\right)$ & 32.3 & 34.1 & 34.9 & $<0.05$ \\
\hline
\end{tabular}

RCT - rotator cuff tears

(as shown in Table 1 of the descriptive statistics). Later we evaluated these parameters and their relationship with RCT. All MRI images of our study were performed by using a 1.5-Tesla unit system (Siemens). A dedicated shoulder array coil was used. When imaging the shoulder with MRI, patients were placed in a supine position with their arms at the sides of their body in partial external rotation with the shoulder coil. Initially, localiser images were obtained, followed by coronal oblique, sagittal oblique, and axial images. The coronal oblique plane was selected parallel to the course of the supraspinatus tendon itself for optimal visualisation of the tendon.

Official permission to carry out the study was obtained from the local medical research Ethics Committee. Written, informed consent was obtained from all study participants.

\section{Statistical analysis}

Data were expressed as mean \pm standard deviation (SD) as well as number and percentage. In addition, Student's $t$-test, the $c^{2}$, and analysis of variance (ANOVA) were used for comparison and correlation of the data. $P<0.05$ was considered statistically significant.

\section{Results}

Of 68 patients with rotator cuff tear, supraspinatus was the most common pathological tendon, with $86 \%$ (59) of cases showing abnormalities. The average age of the patients was
Table 2. Acromial types in different groups

\begin{tabular}{|l|c|c|c|}
\hline Acromial type & $\begin{array}{c}\text { Control group, } \\
n=30\end{array}$ & $\begin{array}{c}\text { Partial RCT, } \\
n=57\end{array}$ & $\begin{array}{c}\text { Complete RCT, } \\
n=11\end{array}$ \\
\hline Type I (flat) & 4 & 5 & 0 \\
\hline Type II (curved) & 19 & 29 & 5 \\
\hline Type III (hooked) & 6 & 21 & 5 \\
\hline Type IV (convex) & 1 & 2 & 1 \\
\hline
\end{tabular}

RCT - rotator cuff tears

$45.11 \pm 21.45$ years with male dominance (80\%). Partial tears of rotator cuff were more common than complete tears. Forty-eight cases showed partial tears in supraspinatus as compared to 11 cases of complete tears. The acromial type and acromial slope did not show any correlation with any particular cuff lesion (as shown in Table 2). AT and AI of controls were significantly smaller than cuff-tear patients. The LAAs of cuff-tear patients differed significantly from those of control patients. The mean acromial thickness in patients with RCT in our study was $8.5 \mathrm{~mm}$.

The impingement patients demonstrated significantly greater acromial thickness, larger CSA and decreased AHD, and decreased LAA compared to their control counterparts (as shown in Table 3).

\section{Discussion}

Identifying the presence of bony impingement of the subacromial space may aid in a better understanding of

Table 3. Comparison of acromial type II and type III in rotator cuff tears (partial and complete) with control group regarding acromial measurements

\begin{tabular}{|l|c|c|c|c|c|}
\hline Acromial measurements & $\begin{array}{c}\text { Type II acromion } \\
\text { (RCT group), } \\
n=34\end{array}$ & $\begin{array}{c}\text { Type III acromion } \\
\text { (RCT group), } \\
n=26\end{array}$ & $\begin{array}{c}\text { Control group } \\
\text { (type II and III), } \\
n=25\end{array}$ & $\begin{array}{c}p \text { value } \\
\text { (type II RCT } \\
\text { vs. control) }\end{array}$ & $\begin{array}{c}p \text { value } \\
\text { (type III RCT } \\
\text { vs. control) }\end{array}$ \\
\hline Acromial thickness (average in $\mathrm{mm}$ ) & 8.6 & 8.9 & 7.9 & $<0.05$ & $<0.001$ \\
\hline Acromiohumeral distance (average in $\mathrm{mm}$ ) & 6.3 & 5.8 & 8.1 & $<0.001$ & $<0.001$ \\
\hline Acromial index (average) & 0.71 & 0.75 & 0.61 & $<0.05$ & $<0.001$ \\
\hline${\text { Lateral acromial angle (average in }{ }^{\circ} \text { ) }}^{\text {(Ritical shoulder angle }}$ & 74.6 & 71.1 & 78.0 & $<0.05$ & $<0.05$ \\
\hline
\end{tabular}

RCT - rotator cuff tears 
predispositions to RC pathology. The factors affecting this space are the type of acromion, which was classified by Bigliani as three types, and later a fourth type was updated. They include type 1 - flat, type II - curved, and type III - hooked acromion morphology, and more recently type IV - convex morphology was added (Table 2) [2].

In the present study, the mean acromion thickness in patients with RCT was significantly thicker than in the control group $(p=0.002)$. This is in accordance with the conclusion of previous studies $[10,11]$; however, there is a slight difference regarding the mean acromial thickness in patients with RCT. It was $8.8 \mathrm{~mm}$ in the study by Paraskevas et al. [10] and $8.3 \mathrm{~mm}$ in the study of Oh et al. [11], but in our study it was $8.5 \mathrm{~mm}$, favouring the previous studies. The presence of os acromiale also decreases the volume of the sub-acromial space and may result in impingement syndromes in some cases. Os acromiale is commonly unilateral and more frequent in type III acromial shape. Detection of the os acromiale on MR images is best achieved by using the axial sequence that includes the acromion [12].

Many studies, which reported no difference in the acromial index of patients who had calcifying tendinitis, partial-thickness rotator cuff tears, or full-thickness rotator cuff tears, called into question the biomechanical theory proposed by Nyffeler et al. regarding the association between large acromial index and rotator cuff disease as well as between small acromial index and osteoarthritis $[9,13,14]$. In contrast, our study supported Nyffeler et al. because a high AI index was seen in RCT patients, especially for full thickness tears [9]. Torrens et al. also reported similar findings in a study in Spain, in which patients with rotator cuff tears had a significantly larger acromial index of 0.72 compared with 0.68 in patients without cuff pathology, and there were differences between the sexes [15]. It was observed that high AI can be one of the associated factors for progression of severity of rotator cuff tears.

One of the other important parameters measured in our study was lateral acromial angle (LAA) on coronal oblique images. In our study, as well as in another by Banas et al., it was found that an extremely low LAA of less than $70^{\circ}$ only occurs in cuff tears. Banas et al. reported that eight patients with complete tears had LAA of less than $70^{\circ}$ and an average age of 54 years, whereas the average age of the remaining 18 patients with cuff tears and LAA greater than $70^{\circ}$ was 70 years [8]. Hence the LAA showed fair correlation with age in our study, and there was moderate correlation ( $\mathrm{PCC}=0.46)$ in the original study by Banas et al. In our opinion, the LAA can help differentiate, on the one hand, between controls and rotator cuff tears and, on the other hand, between impingement and rotator cuff tears.
In our study CSA was found to be 34.6, corroborating with several other authors, such as Gerber et al., Spiegl et al., Moor et al., and Bouaicha et al., who also found similar results [16-19]. Gerber et al. demonstrated a biomechanical study that a high CSA may induce overload of the supraspinatus tendon, particularly at low degrees of active abduction [16]. Garcia et al., in a retrospective study, made a postoperative ultrasound examination after arthroscopic repair of RCTs and found that higher CSA significantly increased the risk of a full thickness rotator cuff re-tear after arthroscopic repair [20]. Although for Kirsch et al. CSA is a strong radiographic predictor of rotator cuff pathology, it did not have a predictive effect on outcomes after arthroscopic repair of atraumatic full-thickness tears [21].

Weiner and Macnab observed that AHD less than $7 \mathrm{~mm}$ is considered proof of a full-thickness tear of the supraspinatus tendon in more than $90 \%$ of cases [22].

Werner et al. believed that the cut-off point for AHD on MRI should be $\leq 6 \mathrm{~mm}$, i.e. lower than on conventional radiograph [23]. In our study the AHD was found to be significantly smaller than in the control group. The present study found a mean subacromial space measurement of $7.71 \mathrm{~mm}$ in the control group, which does not corroborate with the findings of Werner et al., and 6.87 $\mathrm{mm}$ in patients with rotator cuff injuries, favouring many other studies [24-26].

AT, AHD, AI, and LAA were compared between each of the four acromial types in patients with RCT and the control group, and we found that all these acromial measurements were significantly different between only type III acromial shape and the control group. Hence, it could be proposed that it is significantly associated with increased risk of RCT. Paraskevas et al. also studied the clinical significance of the shape of the anterior third of acromion in relation to the impingement syndrome as well as to RCT [10].

\section{Conclusions}

Type 3 acromion is most commonly associated with RCT. The probability of RCT increases if AT $>8.5 \mathrm{~mm}$, LAA $<70^{\circ}, \mathrm{AI}>0.7, \mathrm{CSA}>35^{\circ}$, and $\mathrm{AHD}<7 \mathrm{~mm}$. A better defined acromial morphological criteria should be structured, containing a scoring system from which risk of RCT can be anticipated.

\section{Conflict of interest}

The authors report no conflict of interest. 


\section{References}

1. Mansur DI, Khanal K, Haque MK, et al. Morphometry of acromion process of human scapulae and its clinical importance amongst nepalese population. Kathmandu Univ Med J (KUMJ) 2013; 10: 33-36.

2. Oh JH, Kim JY, Lee HK, et al. Classification and clinical significance of acromial spur in rotator cuff tear: heel-type spur and rotator cuff tear. Clin Orthop Relat Res 2010; 468: 1542-1550.

3. Neer CS 2nd. Impingement lesions. Clin Orthop Relat Res 1983; 173: 70-77.

4. Vitale MA, Arons RR, Hurwitz S, et al. The rising incidence of acromioplasty. J Bone Joint Surg Am 2010; 92: 1842-1850.

5. Bigliani LU, Ticker JB, Flatow EL, et al. The relationship of acromial architecture to rotator cuff disease. Clin Sports Med 1991; 10 823-838.

6. Kitay GS, Iannotti JP, Williams GR, et al. Roentgenographic assess ment of acromial morphologic condition in rotator cuff impingement syndrome. J Shoulder Elbow Surg 1995; 4: 441-448.

7. Aoki M, Ishii S, Usui M. The slope of the acromion and rotator cuff impingement. Orthop Trans 1986; 10: 228.

8. Banas MP, Miller RJ, Totterman S. Relationship between the lateral acromion angle and rotator cuff disease. J Shoulder Elbow Surg 1995; 4: 454-461.

9. Nyffeler RW, Werner CM, Sukthankar A, et al. Association of a large lateral extension of the acromion with rotator cuff tears. J Bone Joint Surg Am 2006; 88: 800-805.

10. Paraskevas G, Tzaveas A, Papaziogas B, et al. Morphological parameters of the acromion. Folia Morphol 2008; 67: 255-260.

11. Oh JH, Kim JY, Lee HK, et al. Classification and clinical significance of acromial spur in rotator cuff tear: heel-type spur and rotator cuff tear. Clin Orthop Relat Res 2010; 468: 1542-1550.

12. Wright R, Heller M, Quick D, et al. Arthroscopic decompression for impingement syndrome secondary to an unstable os acromiale. Arthroscopy 2000; 16: 595-599.

13. Miyazaki A, Itoi E, Sano H. Comparison between the acromion index and rotator cuff tears in the Brazilian and Japanese populations. J Shoulder Elbow Surg 2011; 20: 1082-1086.

14. Kappe T, Cakir B, Lippacher S, et al. Intraarticular lesions in calcifying tendinitis: incidence and association with the acromion index. Arch Orthop Trauma Surg 2011; 131: 325-329.
15. Torrens C, Lopez JM, Puente I, et al. The influence of the acromial coverage index in rotator cuff tears. J Shoulder Elbow Surg 2007; 16: $347-351$

16. Gerber C, Snedeker JG, Baumgartner D, et al. Supraspinatus tendon load during abduction is dependent on the size of the critical shoulder angle: a biomechanical analysis. J Orthop Res 2014; 32: 952-957.

17. Spiegl UJ, Horan MP, Smith SW, et al. The critical shoulder angle is associated with rotator cuff tears and shoulder osteoarthritis and is better assessed with radiographs over MRI. Knee Surg Sports Traumatol Arthrosc 2016; 24: 2244-2251

18. Moor BK, Röthlisberger M, Müller DA, et al. Age, trauma and the critical shoulder angle accurately predict supraspinatus tendon tears. Orthop Traumatol Surg Res 2014; 100: 489-494.

19. Bouaicha S, Ehrmann C, Slankamenac K, et al. Comparison of the critical shoulder angle in radiographs and computed tomography. Skeletal Radiol 2014; 43: 1053-1056.

20. Garcia GH, Liu JN, Degen RM, et al. Higher critical shoulder angle increases the risk of retear after rotator cuff repair. J Shoulder Elbow Surg 2017; 26: 241-245.

21. Kirsch JM, Nathani A, Robbins CB, et al. Is there an association between the "critical shoulder angle" and clinical outcome after rotator cuff repair? Orthop J Sports Med 2017; 5: 2325967117702126.

22. Weiner DS, Macnab I. Superior migration of the humeral head. A radiological aid in the diagnosis of tears of the rotator cuff. J Bone Joint Surg Br 1970; 52: 524-527.

23. Werner CM, Conrad SJ, Meyer DC, et al. Intermethod agreement and interobserver correlation of radiologic acromiohumeral distance measurements. J Shoulder Elbow Surg 2008; 17: 237-240.

24. Saupe N, Pfirrmann CW, Schmid MR, et al. Association between rotator cuff abnormalities and reduced acromiohumeral distance. AJR Am J Roentgenol 2006; 187: 376-382.

25. Balke M, Schmidt C, Dedy N, et al. Correlation of acromial morphology with impingement syndrome and rotator cuff tears. Acta Orthop 2013; 84: 178-183.

26. Li X, Xu W, Hu N, et al. Relationship between acromial morphological variation and subacromial impingement: a three-dimensional analysis. PLoS One 2017; 12: e0176193. 